\title{
Sequence Analysis: Time Use Data (ATUS)
}

\author{
Kamila Kolpashnikova*
}

\section{Introduction}

Traditionally, a tool for analysing life events in social sciences, sequence analysis is becoming more and more popular for dealing with time-use data. In this tutorial, I'll walk you through the steps how to do sequence analysis with time-use data (American Time Use Survey, ATUS).

To follow this tutotial:

- change the directory to the location of cloned directory

- follow instructions in the code (R file is included)

Funding: This project has received funding from the European Union's Horizon 2020 research and innovation programme under the Marie Sklodowska-Curie grant agreement No 892101 (awardee: Kamila Kolpashnikova).

\section{Example of Work Using Sequence Analysis}

Here is an example how a published paper using sequence analysis looks like (my recent research in the Journal of Population Ageing):

Kolpashnikova, K., \& Kan, M. Y. (2020). Eldercare in Japan: Cluster Analysis of daily time-use patterns of elder caregivers. Journal of Population Ageing, 1-23.

If you follow this tutorial, you will be able to recreate the analyses in the paper.

\section{Loading Necessary Packages}

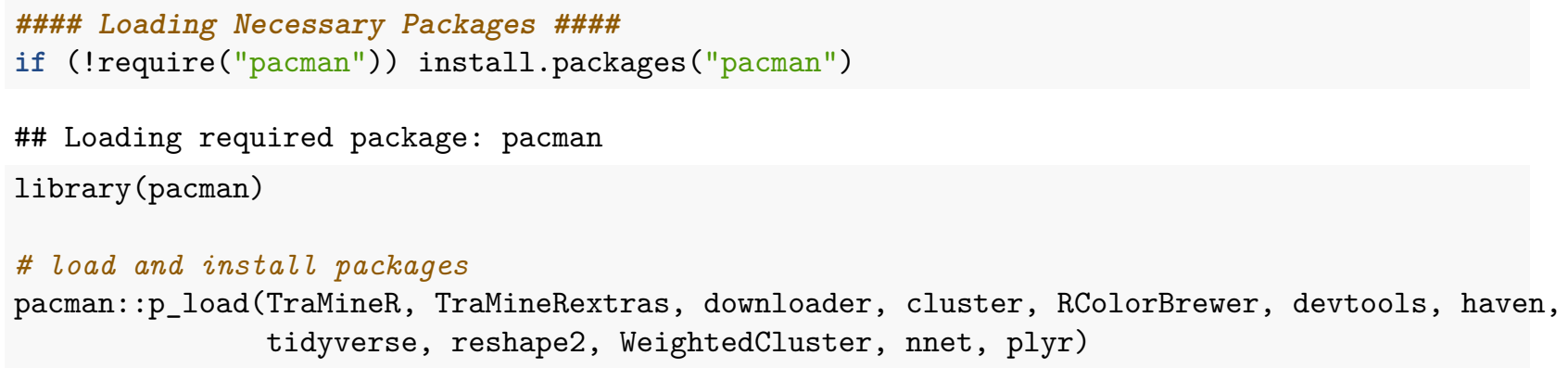

\section{Load Datasets from the BLS website}

Remember that in r, it's forward slashes. Here I created a function that can unzip and create dataframes from the zipped BLS ATUS files.

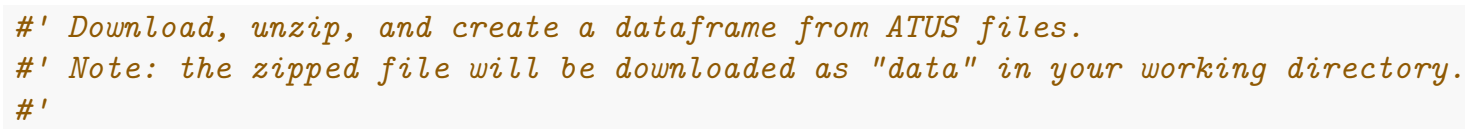

*University of Oxford 


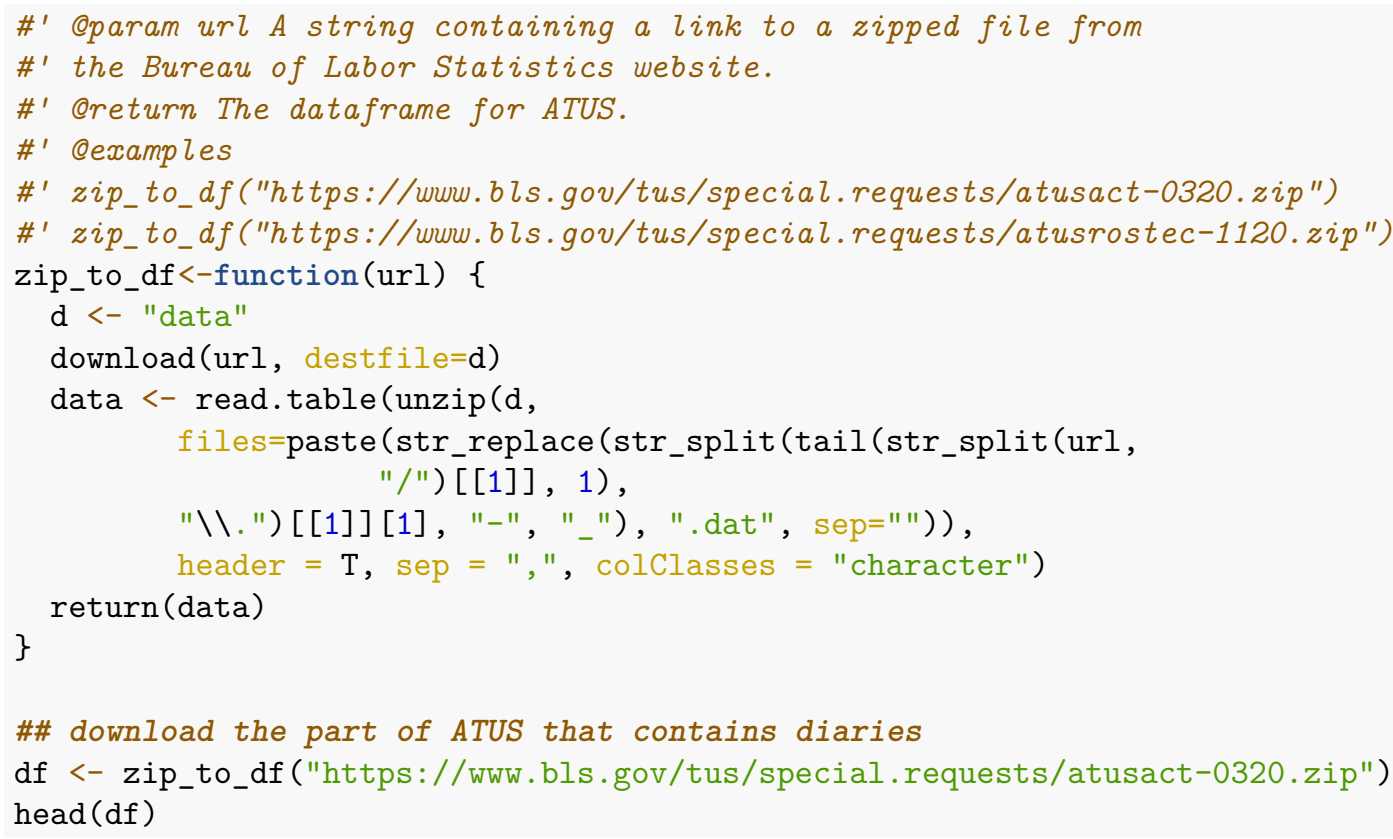

\#\# download the part of ATUS with the information about self-reported elder caregivers df_ec <- zip_to_df("https://www.bls.gov/tus/special.requests/atusrostec-1120.zip") head (df_ec) 


$\begin{array}{llllllllll}\text { \#\# } & 1 & 20110101110074 & 70 & 4 & 44 & 2 & 0 & 5 & -1 \\ \text { \#\# } & 2 & 20110101110156 & 85 & 4 & 46 & 2 & 0 & 5 & -1 \\ \text { \#\# } & 3 & 20110101110507 & 80 & 1 & 55 & -1 & 0 & 2 & -1 \\ \text { \#\# } & 4 & 20110101110521 & 85 & 3 & 43 & -1 & 0 & 3 & -1 \\ \text { \#\# } & 5 & 20110101110522 & 80 & 4 & 44 & 6 & 0 & 2 & -1 \\ \text { \#\# } & 6 & 20110101110639 & 64 & 1 & 38 & -1 & 0 & 2 & -1\end{array}$

\section{Filter the elder caregivers and recode activities}

Before we create our sequence dataframes, we need to wrangle the original data to make it usable.

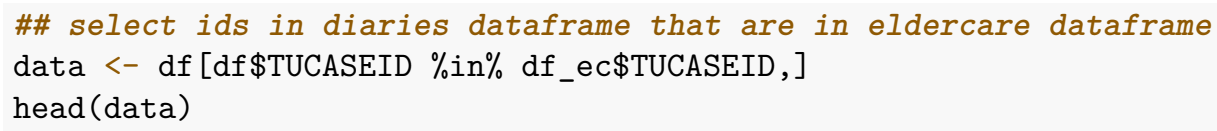




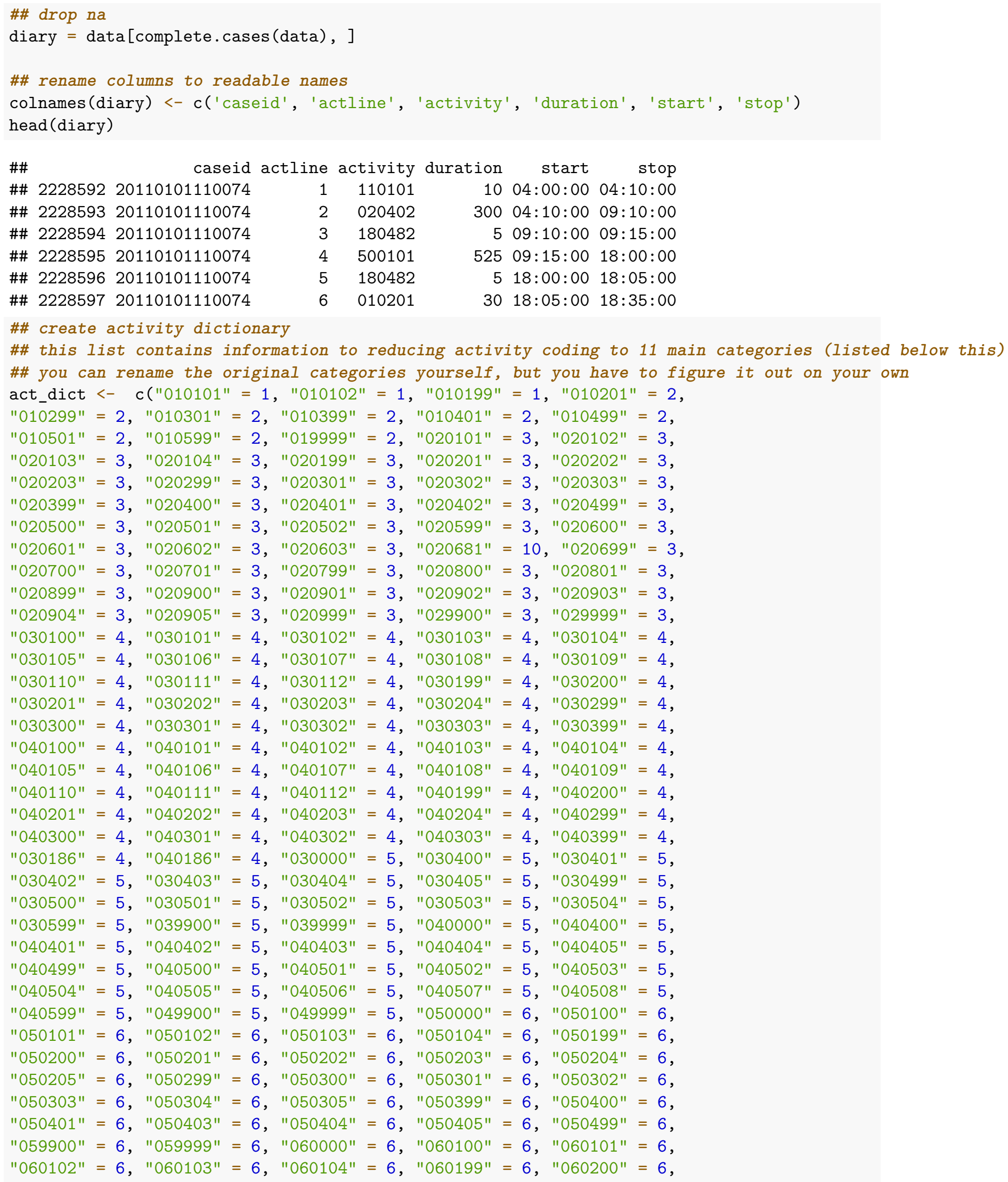




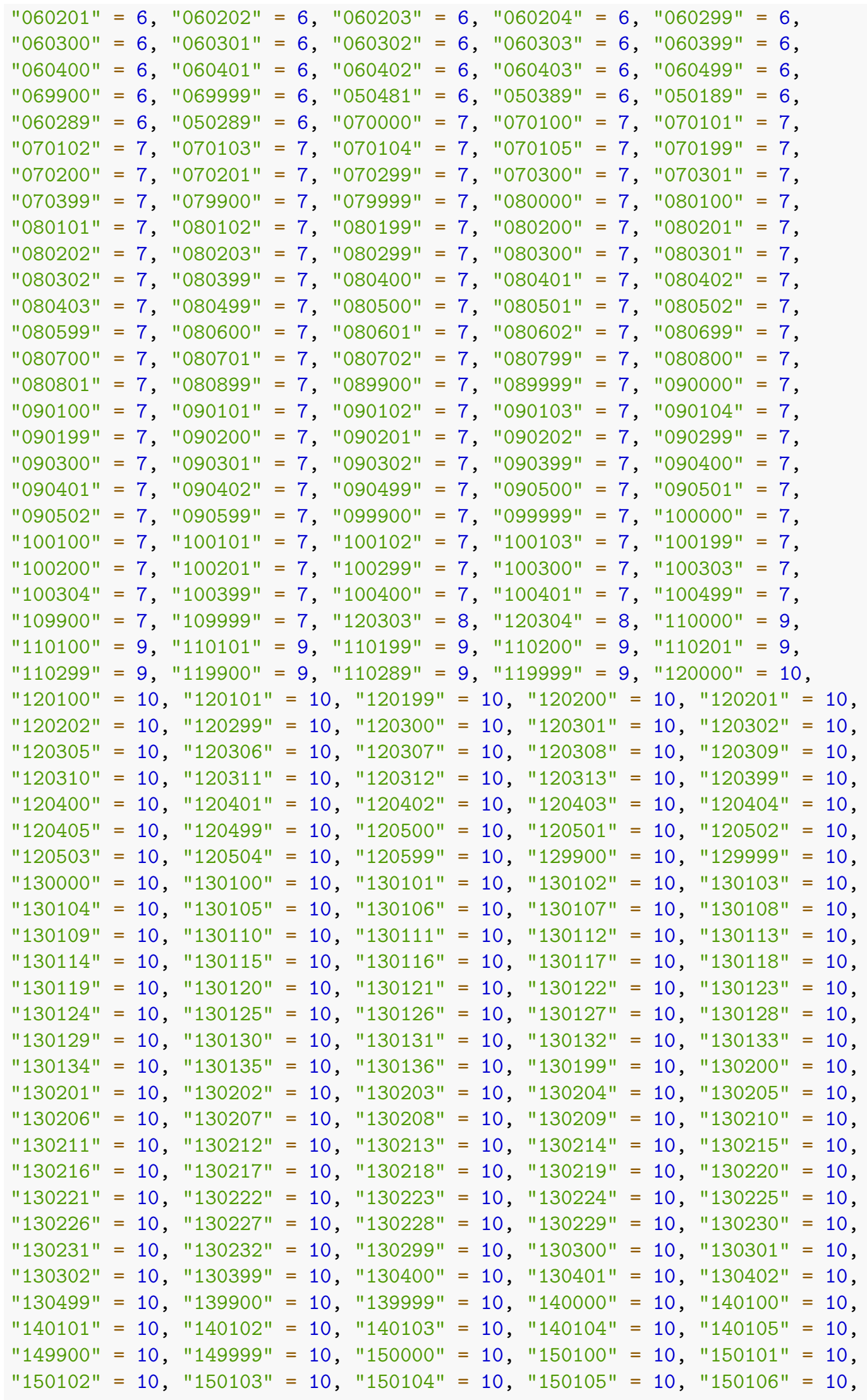




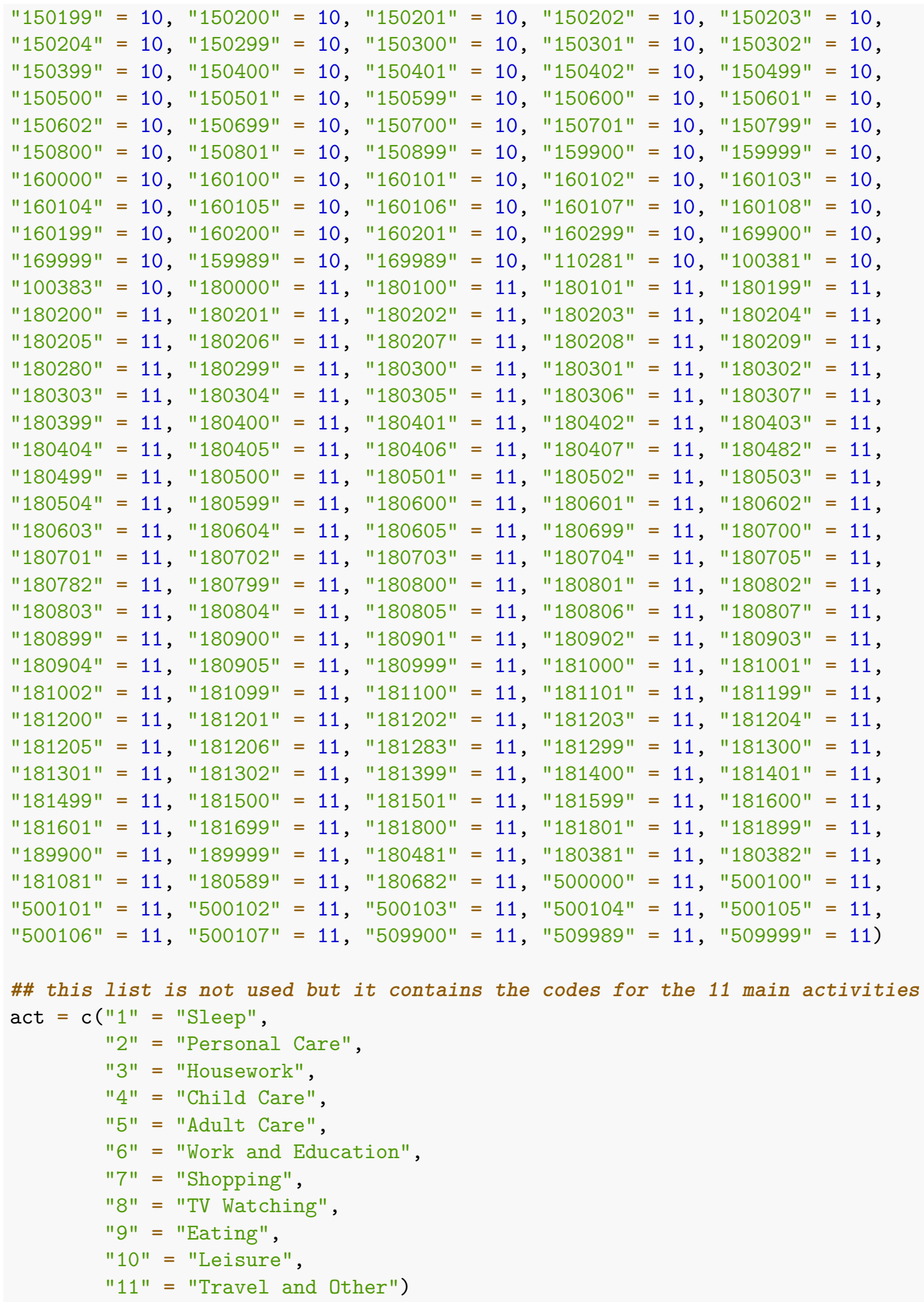

\#\#\# recode the activities using the activity dictionary diary\$lst_act <- revalue(diary\$activity, act_dict)

\#\# The following 'from' values were not present in 'x': 010199, 010599, 019999, 020400, 020500, 020600, 


\section{Sequence Analysis}

I create an object with intervals' labels. Sequences start at 04:00 AM: Depending on your own sequence intervals (if you are not using the ATUS) these labels need to be adjusted

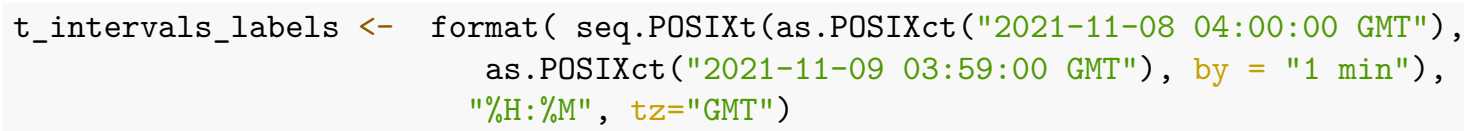

\section{Colour Palette}

In my experience, brewing colours in $\mathrm{R}$ is one of the most painful experiences, especially if you have many categories of activities. Things can get even worse if you have to go grayscale if publishing articles with colour images is too expensive.

Let's brew some colours first.The number of colours is dictated by the number of states (in the alphabet). We reduced it to 8, so it won't create difficulties (difficulties arise when there are more than 12 categories).

Interesting resource on colors (cheatsheet):

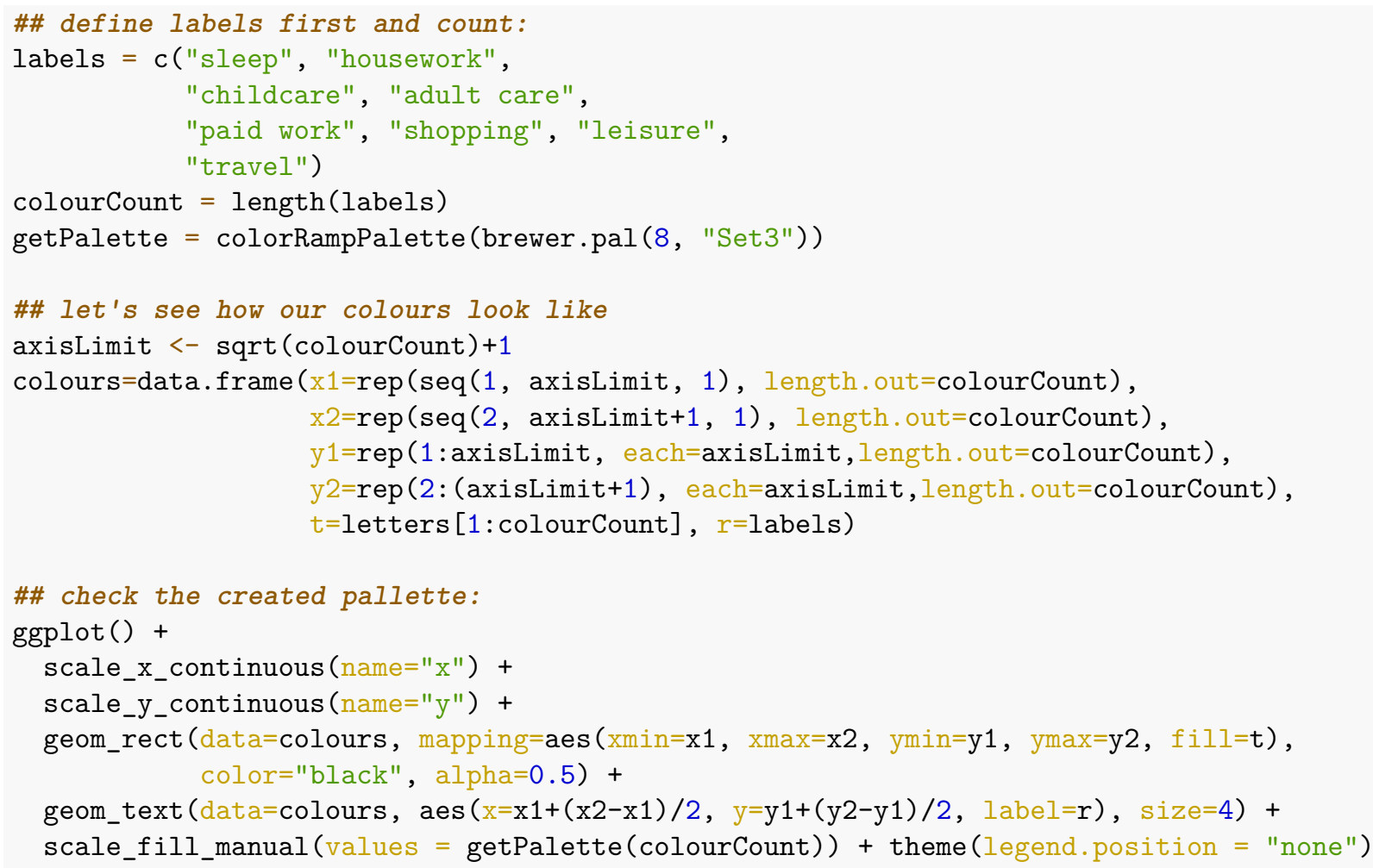




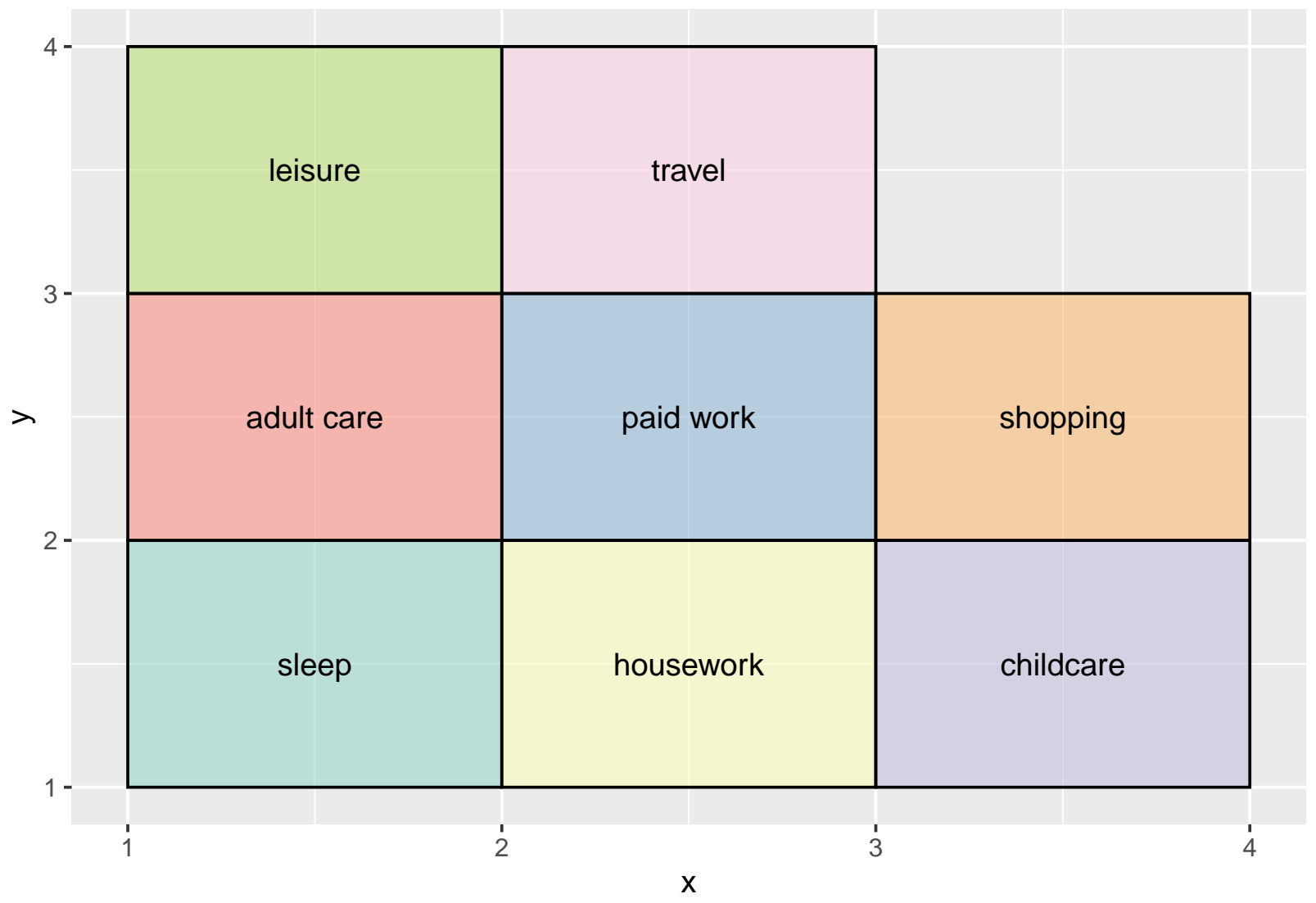

\section{Defining Sequence Object}

Before defining a sequence object, I reduced the dataframe to 2000 sequences. This is because I wanted for the tutorial demostration to run faster. You can use all sequences as long as they are less than about 46000 in total. On my laptop 46000's dissimilarity matrix calculation ran for 4 hours but the cluster analysis crushed. So, I would recommend to use a random smaller sample if you have huge ones. Or, run the analyses on chunks and then combine the similar-looking clusters.

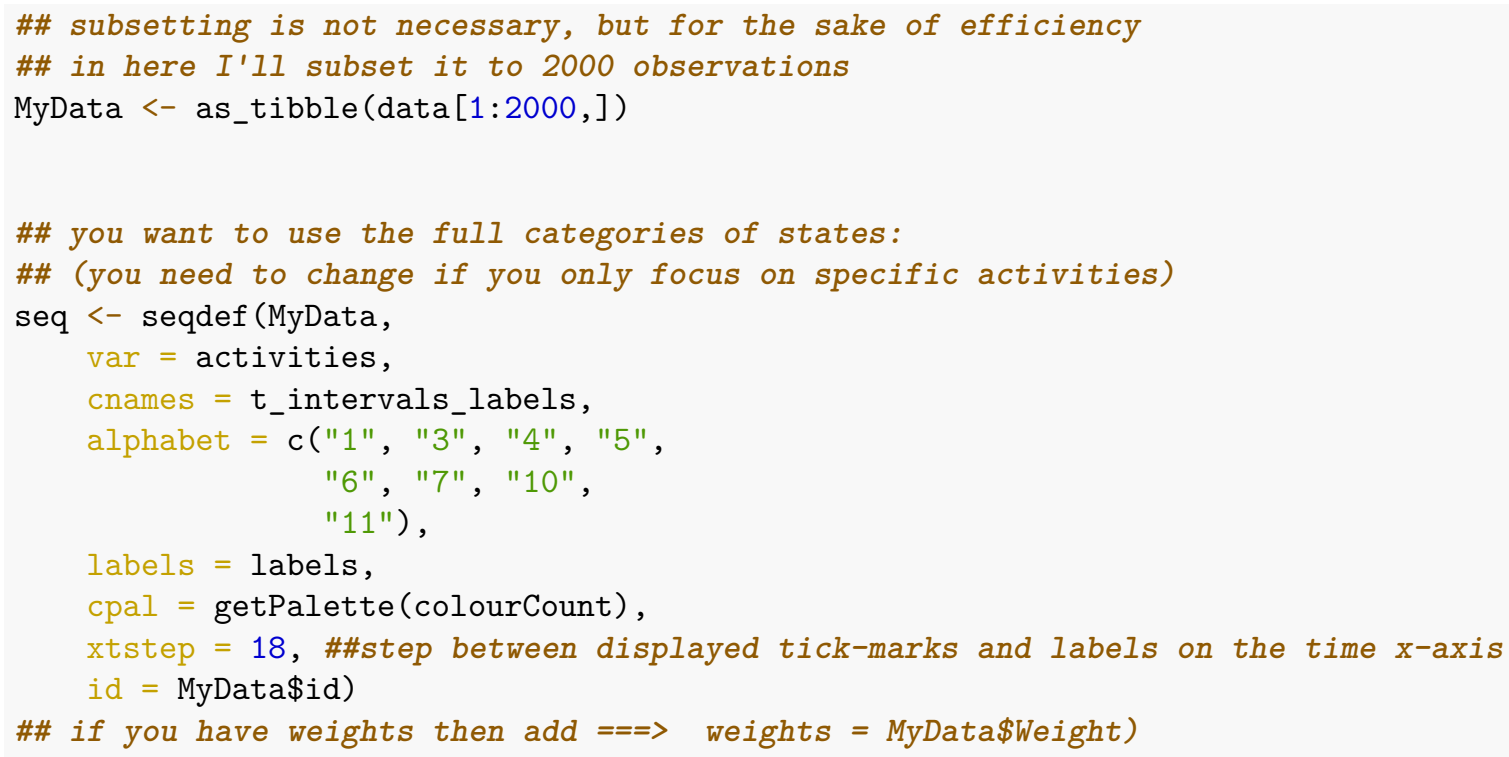


\#\# check how the sequence looks like

$\operatorname{print}(\operatorname{seq}[1: 5$,$] , format =$ "STS")

\#\# Sequence

\#\# 20110101110074 10-10-10-10-10-10-10-10-10-10-3-3-3-3-3-3-3-3-3-3-3-3-3-3-3-3-3-3-3-3-3-3-3-3-3-3-3-3

\#\# 20110101110156 1-1-1-1-1-1-1-1-1-1-1-1-1-1-1-1-1-1-1-1-1-1-1-1-1-1-1-1-1-1-1-1-1-1-1-1-1-1-1-1-1-1-1

\#\# 20110101110507 1-1-1-1-1-1-1-1-1-1-1-1-1-1-1-1-1-1-1-1-1-1-1-1-1-1-1-1-1-1-1-1-1-1-1-1-1-1-1-1-1-1-1

\#\# 20110101110521 10-10-10-10-10-10-10-10-10-10-10-10-10-10-10-10-10-10-10-10-10-10-10-10-10-10-10-10-1

\#\# 20110101110522 1-1-1-1-1-1-1-1-1-1-1-1-1-1-1-1-1-1-1-1-1-1-1-1-1-1-1-1-1-1-1-1-1-1-1-1-1-1-1-1-1-1-1

\#\# "STS" format shows each step

\section{Plotting Sequences}

- sequence index plots

With a small $\mathrm{i}$, the default for idxs is $1: 10$, plotting the first 10 sequences. If you set idxs to 0 , it plots all sequences (might take a long time).

seqiplot (seq, border $=$ NA, with.legend $=$ "right", legend.prop=0.4)

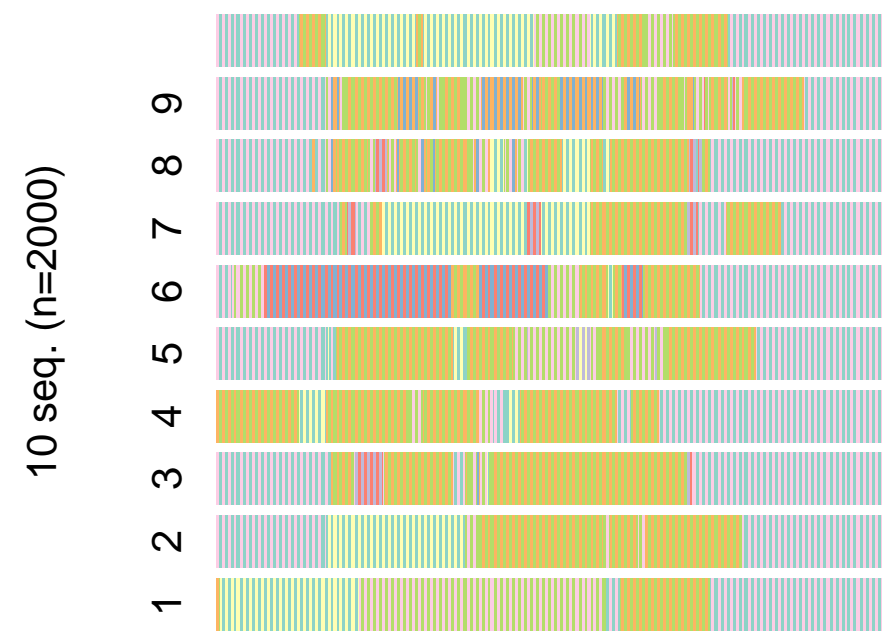

sleep

housework

childcare

adult care

paid work

shopping

leisure

travel

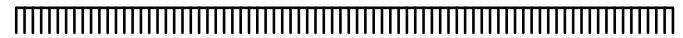

\section{4:00 $\quad 09: 06 \quad 14: 12 \quad 19: 18 \quad 00: 24$}

You can also use the same command with a capital I. It wiil plot all unless you specify idxs option.

seqIplot (seq, border $=$ NA, with.legend = "right", legend.prop=0.4, idxs = 1:4) 


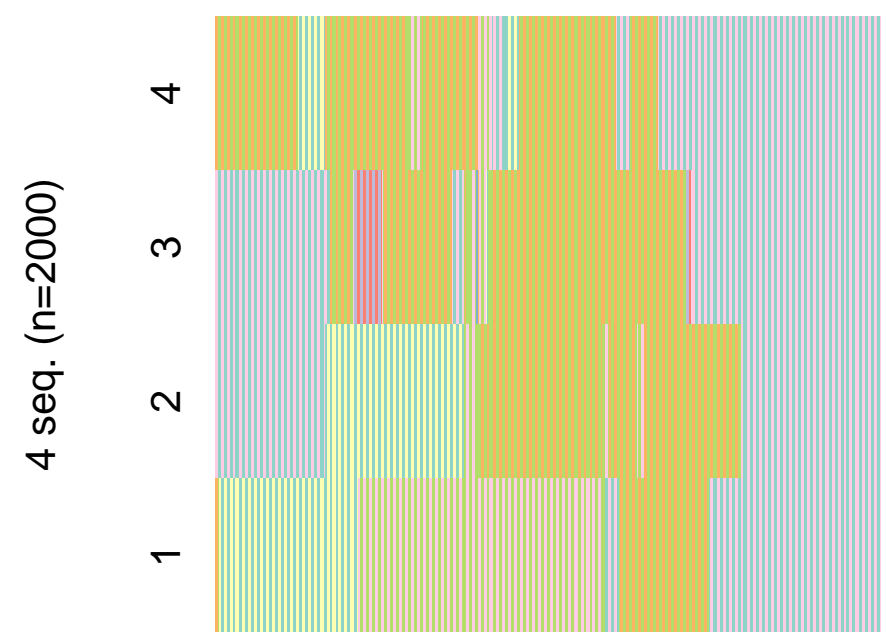

\begin{tabular}{|ll|}
\hline$\square$ & sleep \\
$\square$ & housework \\
$\square$ & childcare \\
$\square$ & adult care \\
$\square$ & paid work \\
$\square$ & shopping \\
$\square$ & leisure \\
$\square$ & travel \\
\hline
\end{tabular}

|ा|ा|ा|ा|ा|ा|ा|ा|ा|ा|ा|ा|ा|ा|ा|ा|ा|ा|ा|ा|ा|ा|ा|ा|ा|ा|ा|ा|ा|ा|ा|ा|ा|ा|ा|ा|

04:00 $\quad 09: 06 \quad 14: 12 \quad 19: 18 \quad 00: 24$

- the most frequent sequences

Unfortunately, for time-use data, it is usually useless to plot the most frequent sequences. If you tabulate 4 frequent sequences you will see what I mean. It is because there are 1440 steps and there are barely any same sequences. This command is more useful for shorter sequences with many commonalities (as in life-course research).

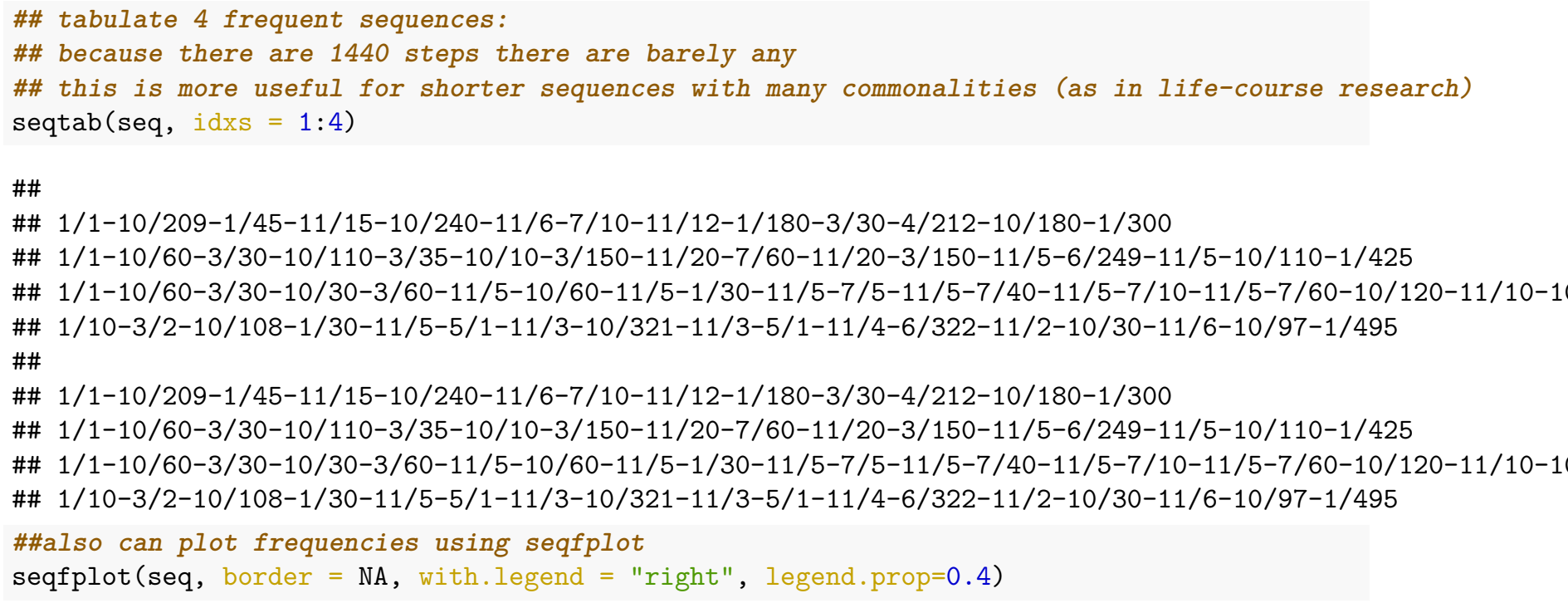




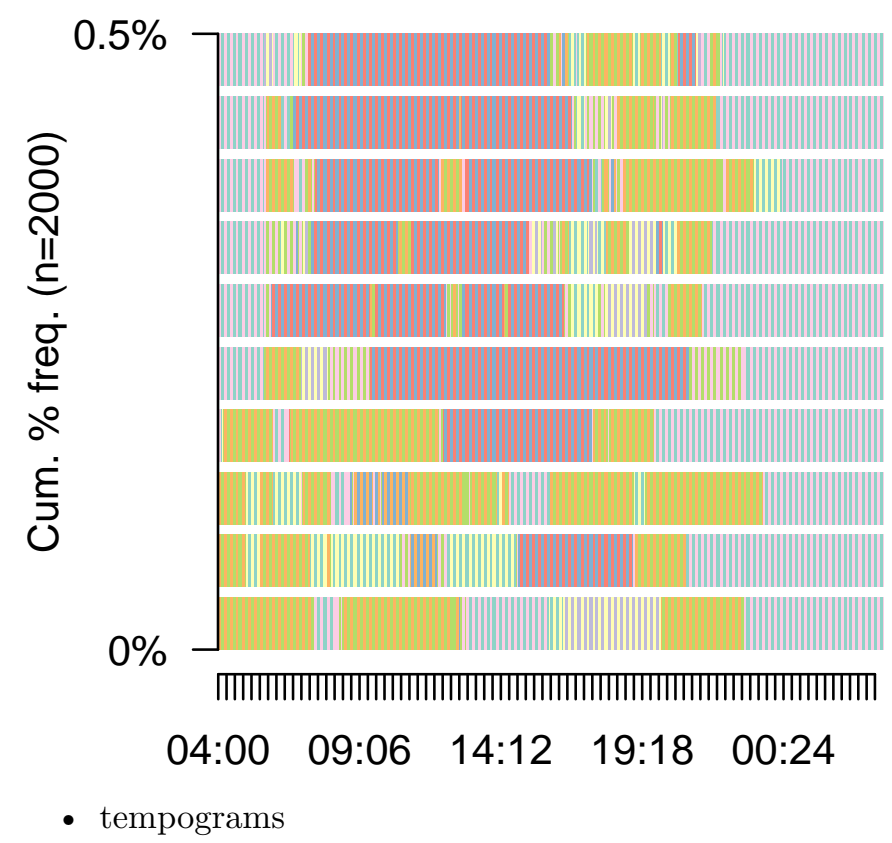

\begin{tabular}{|l|}
\hline$\square$ sleep \\
$\square$ housework \\
$\square$ childcare \\
$\square$ adult care \\
$\square$ paid work \\
$\square$ shopping \\
$\square$ leisure \\
$\square$ travel \\
\hline
\end{tabular}

State distribution plots (aka tempogram aka chronogram) This is an easy way to plot a tempogram (compared to area plots).

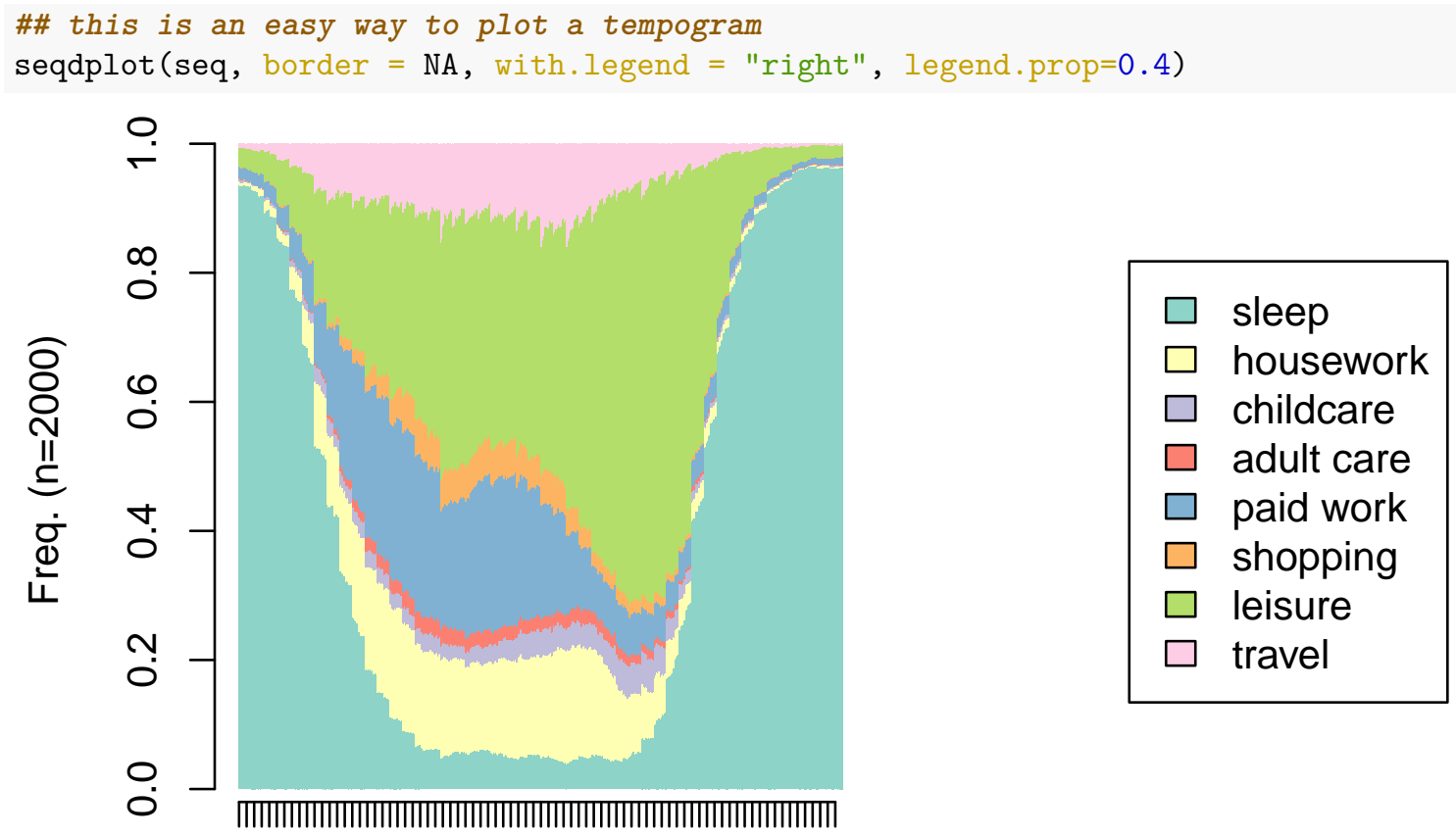

$\begin{array}{lllll}04: 00 & 09: 42 & 15: 24 & 21: 06 & 02: 48\end{array}$

\section{Transitions}

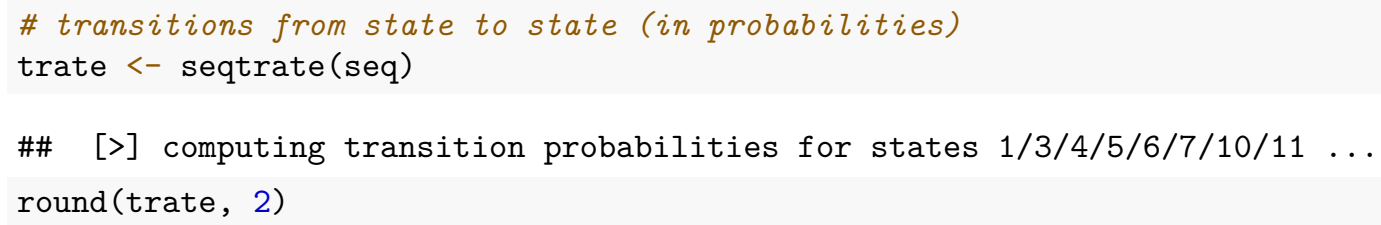




\begin{tabular}{|c|c|c|c|c|c|c|c|c|}
\hline \# & 1] & 3] & $\rightarrow$ & 年 & {$[->$} & 7] & & \\
\hline$\# \#\left[\begin{array}{ll}1 & ->\end{array}\right]$ & 1 & 0.00 & 0.00 & 0.00 & 0.00 & 0.00 & 0.00 & 0.00 \\
\hline$\#\left[\begin{array}{ll}3 & ->\end{array}\right]$ & 0 & 0.98 & 0.00 & 0.00 & 0.00 & 0.00 & 0.01 & 0.00 \\
\hline$\# \#\left[\begin{array}{ll}4 & ->\end{array}\right]$ & 0 & 0.00 & 0.97 & bo & 0. & 0 & .01 & 0.01 \\
\hline $5->]$ & 0 & 0.00 & 0.00 & .98 & 0.00 & 0 & 0.00 & 0.01 \\
\hline$\# \#\left[\begin{array}{ll}6 & ->\end{array}\right]$ & 0 & 0.00 & 0.00 & .00 & 0. & 0 & .00 & 0.00 \\
\hline$\# \#\left[\begin{array}{ll}7 & ->\end{array}\right]$ & 0 & 0.00 & 0.00 & .00 & 0.00 & 0.97 & 0.00 & 0.03 \\
\hline \#\# [10 -> $]$ & 0 & 0.00 & 0.00 & 0.00 & 0.00 & 0.00 & 0.99 & 0.00 \\
\hline$\#\left[\begin{array}{ll}11 & ->\end{array}\right]$ & 0 & 0.01 & 0.00 & 0.00 & 0.00 & 0.01 & 0.02 & 0.9 \\
\hline
\end{tabular}

\#\# heatmap of the transitions matrix heatTrate=melt (trate) head (heatTrate)
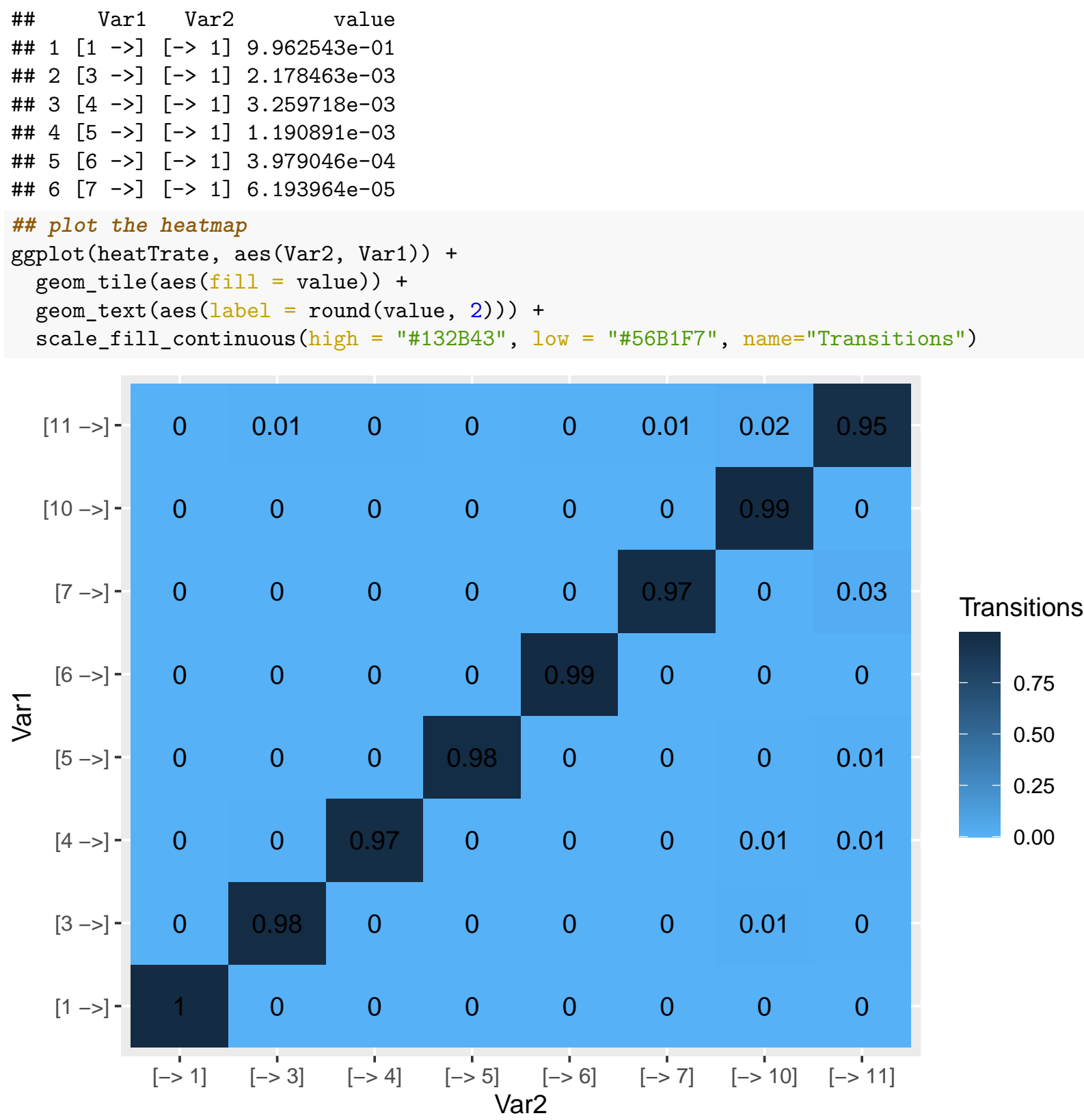


\section{Changing Granularity (Minutes to Hours, etc.)}

TraMineR made it very easy to change the number of steps in a sequence. seqgranularity is the command that will help you do it.

To use the first state to represent all, use method = "first", the last = "last", or the most frequent = "mostfreq".

In the following chunk of code, tspan $=15$ means to transform the step to every 15 min (instead of every minute).

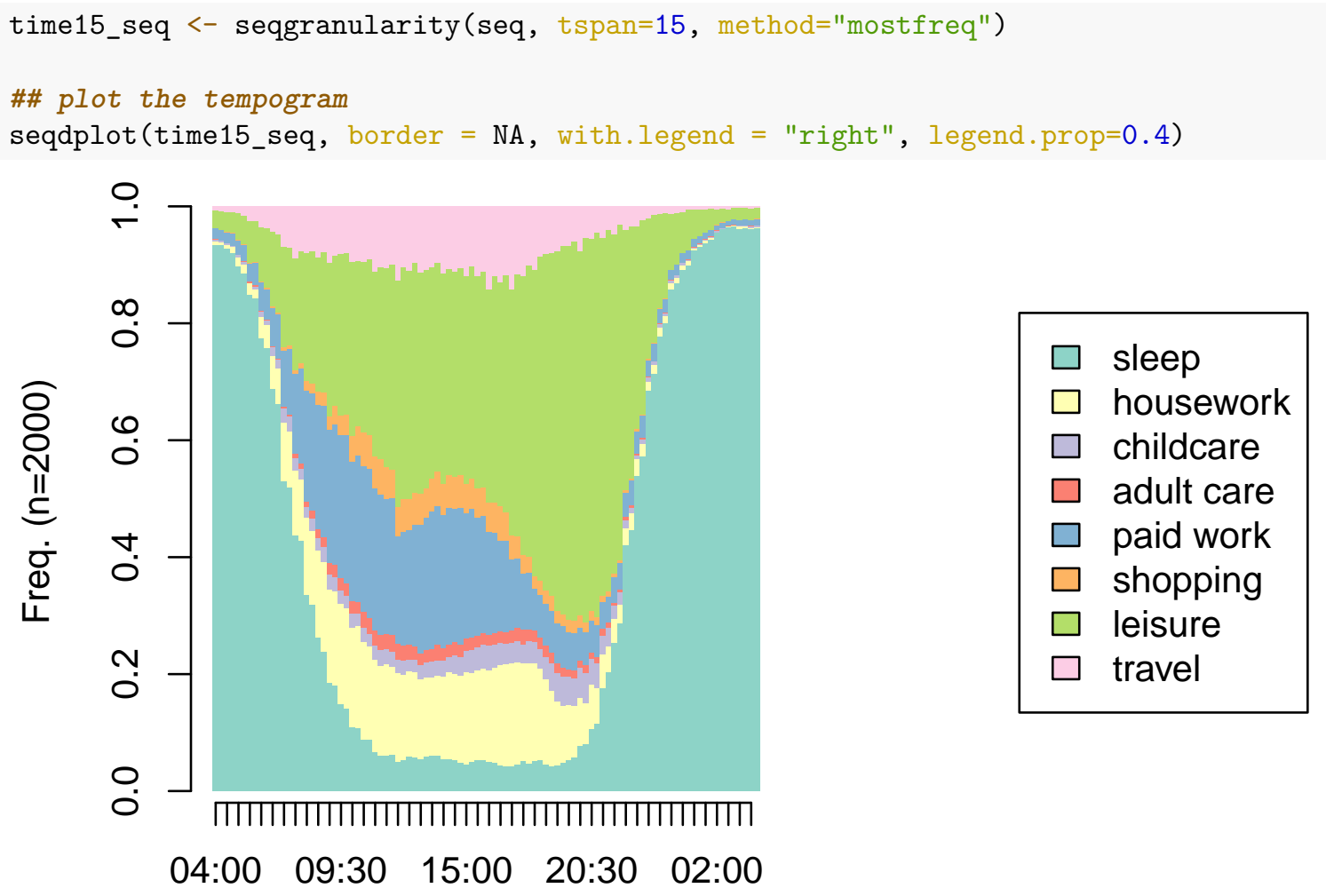

You can see on the tempograms that the granularity decreased and now each step is a 15-minute slot.

\section{Modal states sequence}

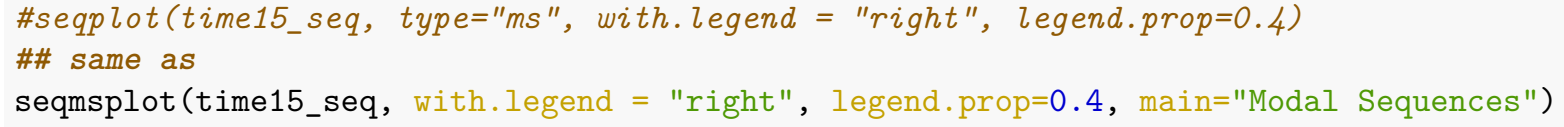




\section{Modal Sequences}

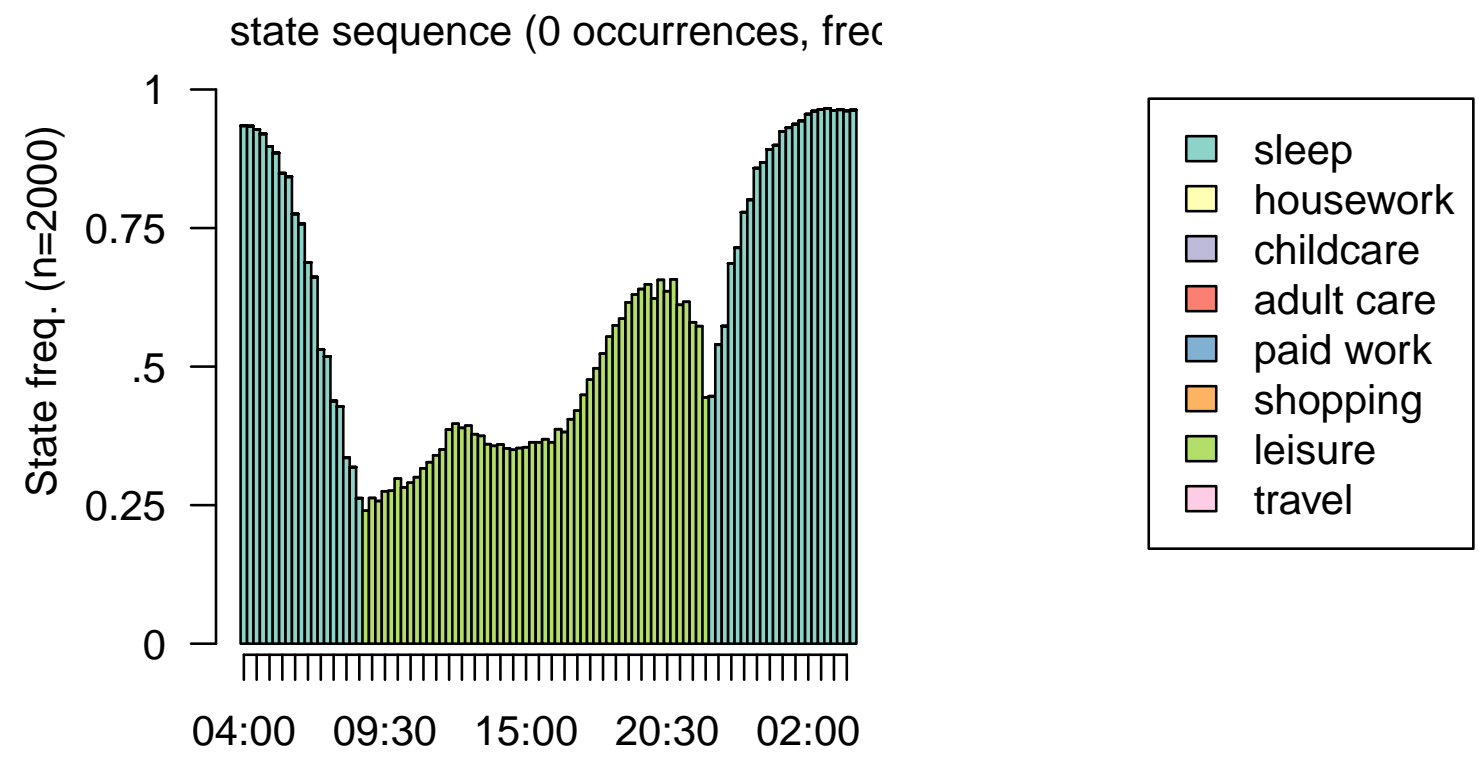

\section{Embrace entropy}

The higher the value, the more diverse are activities at that time.

\#transversal entropy of state distributions

\#the number of valid states and the Shannon entropy of the transversal state distribution \# shows the measure of 'chaos' (diversity of activities) in the diaries

seqHtplot(time15_seq, with.legend = "right", legend.prop=0.4)

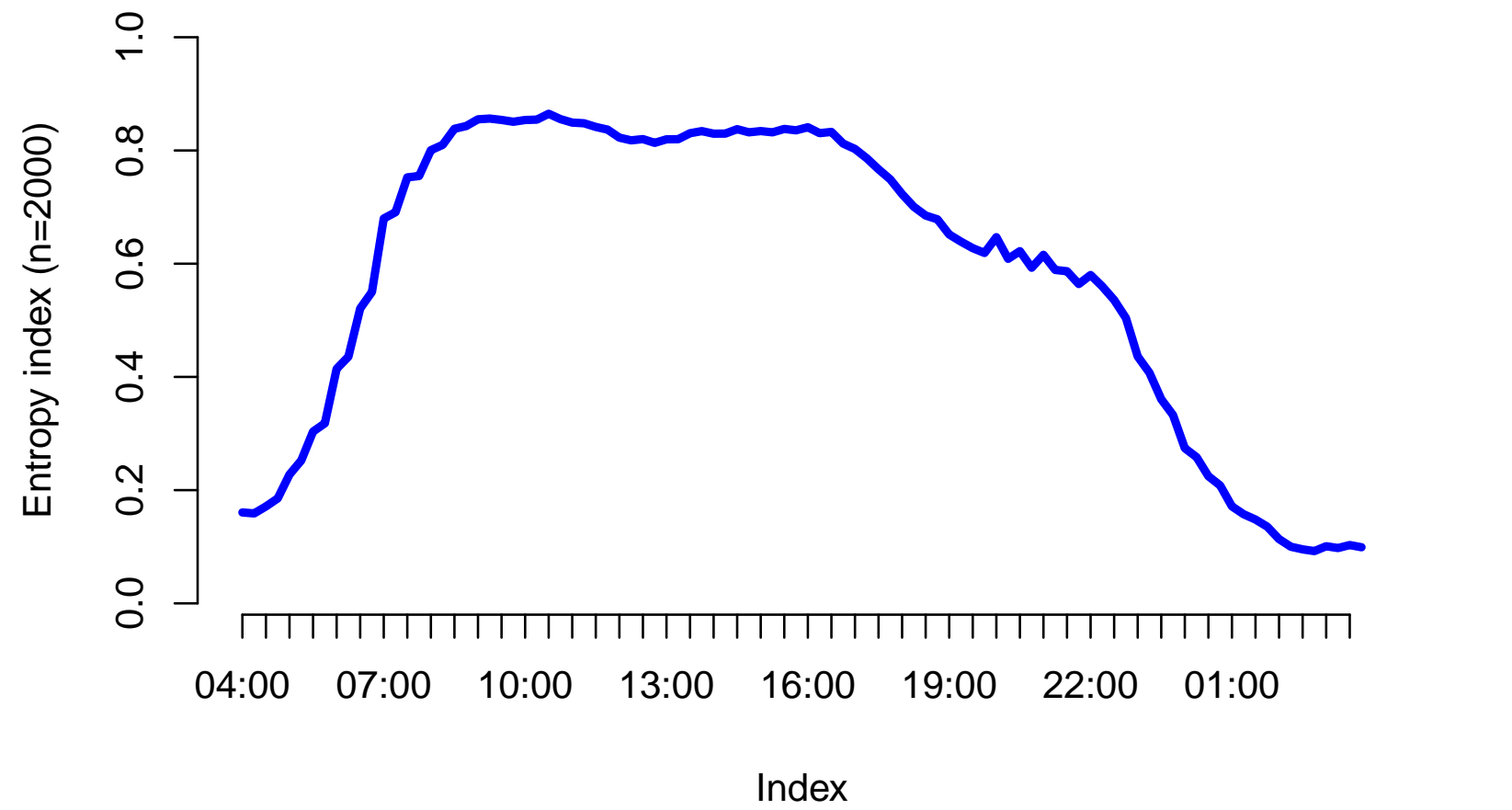




\section{Calculating Dissimilarities}

\section{Substitution Cost Matrix}

We need to define the substitution cost for all the transitions (it can be a constant or user-defined).

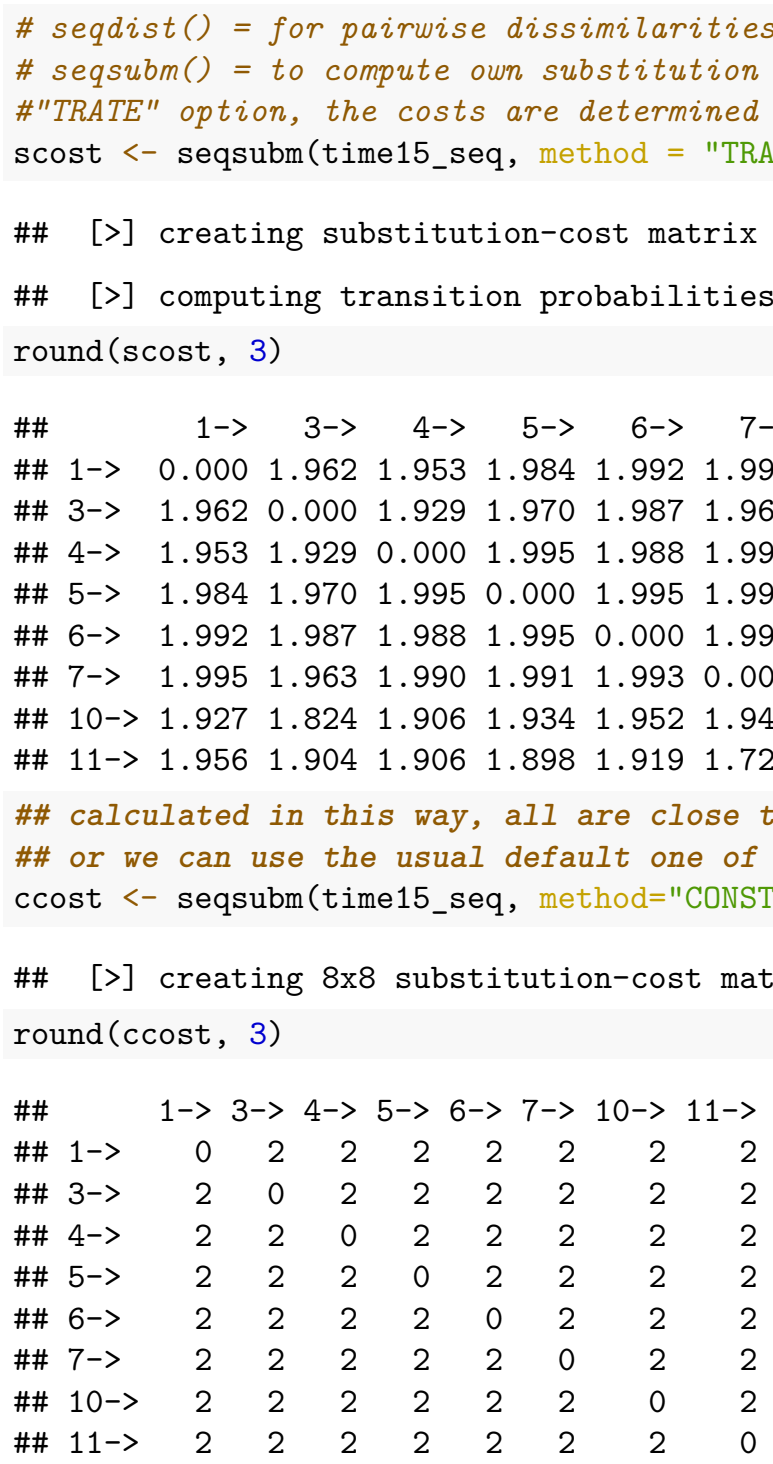

\section{Optimal Matching}

Optimal matching for calculating dissimilarities between sequences need the specification of both substitution and indel costs. The algorithm is developed by Needleman and Wunsch (1970). For the illustration how the algorithm works please link to my explanation of optimal matching

If the sequence file is heavy, calculate only the upper part of the matrix by full.matrix = FALSE The usual default is that substitution cost is twice the indel cost, and default indel cost is 1 .

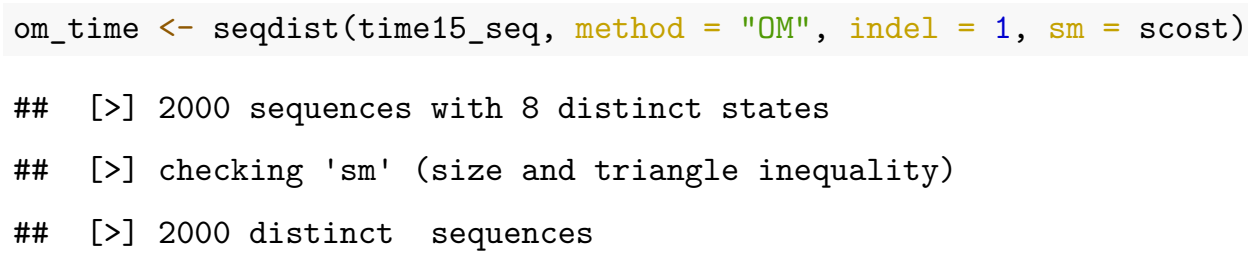


\#\# [>] min/max sequence lengths: 96/96

\#\#[>] computing distances using the OM metric

\#\# [>] elapsed time: 28.864 secs

\#\# this results in a dissimilarity matrix which you can look at using: round (om_time $[1: 10,1: 10], 1$ )

$\begin{array}{rrrrrrrrrrrr}\# \# & & {[, 1]} & {[, 2]} & {[, 3]} & {[, 4]} & {[, 5]} & {[, 6]} & {[, 7]} & {[, 8]} & {[, 9]} & {[, 10]} \\ \# \# & {[1,]} & 0.0 & 72.6 & 102.9 & 87.5 & 86.5 & 93.1 & 86.3 & 95.7 & 106.2 & 63.8 \\ \# \# & {[2,]} & 72.6 & 0.0 & 46.4 & 67.4 & 48.6 & 102.1 & 46.0 & 49.1 & 70.4 & 53.0 \\ \# \# & {[3,]} & 102.9 & 46.4 & 0.0 & 51.2 & 39.5 & 93.8 & 72.9 & 36.1 & 72.7 & 81.3 \\ \# \# & {[4,]} & 87.5 & 67.4 & 51.2 & 0.0 & 64.2 & 93.7 & 99.0 & 63.4 & 100.3 & 88.5 \\ \# \# & {[5,]} & 86.5 & 48.6 & 39.5 & 64.2 & 0.0 & 97.3 & 73.2 & 50.3 & 58.3 & 66.0 \\ \# \# & {[6,]} & 93.1 & 102.1 & 93.8 & 93.7 & 97.3 & 0.0 & 116.3 & 86.9 & 111.2 & 100.6 \\ \# \# & {[7,]} & 86.3 & 46.0 & 72.9 & 99.0 & 73.2 & 116.3 & 0.0 & 66.4 & 87.1 & 45.1 \\ \# \# & {[8,]} & 95.7 & 49.1 & 36.1 & 63.4 & 50.3 & 86.9 & 66.4 & 0.0 & 66.4 & 68.0 \\ \# \# & {[9,]} & 106.2 & 70.4 & 72.7 & 100.3 & 58.3 & 111.2 & 87.1 & 66.4 & 0.0 & 87.9 \\ \# \# & {[10,]} & 63.8 & 53.0 & 81.3 & 88.5 & 66.0 & 100.6 & 45.1 & 68.0 & 87.9 & 0.0\end{array}$

\section{Cluster Analysis}

Let's run cluster analysis on our dissimilarity matrix

Other common methods are:

- "average",

- "single",

- "complete"

The "average" and "single" options do not work well for time-use data (check). The "complete" option is a possibility (check).

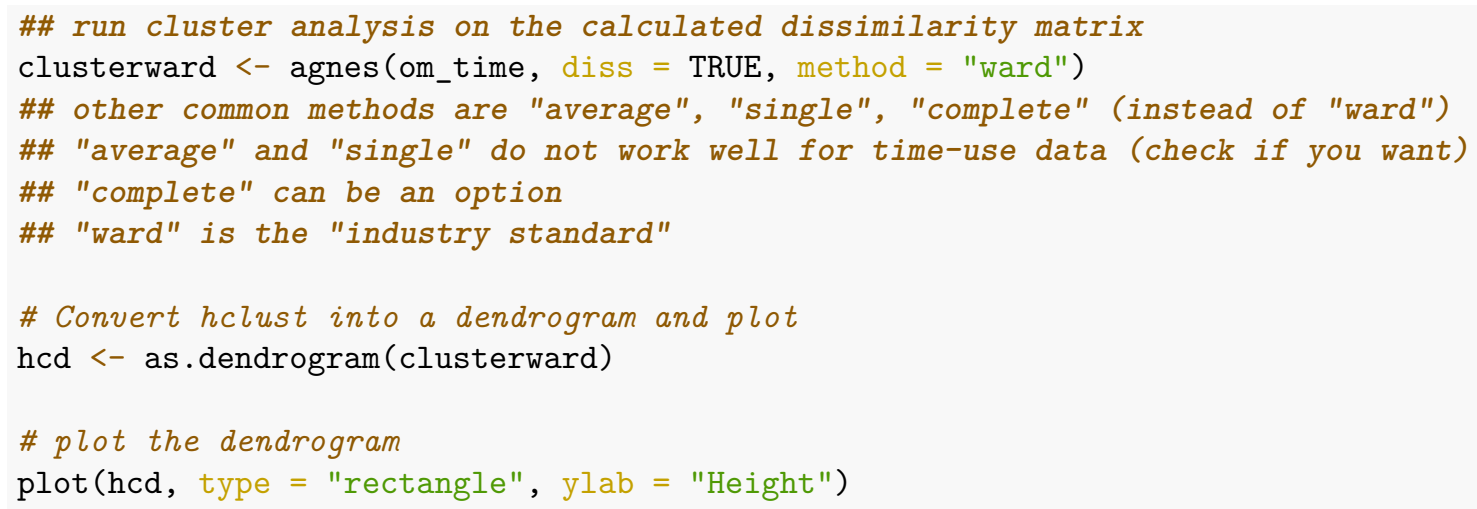




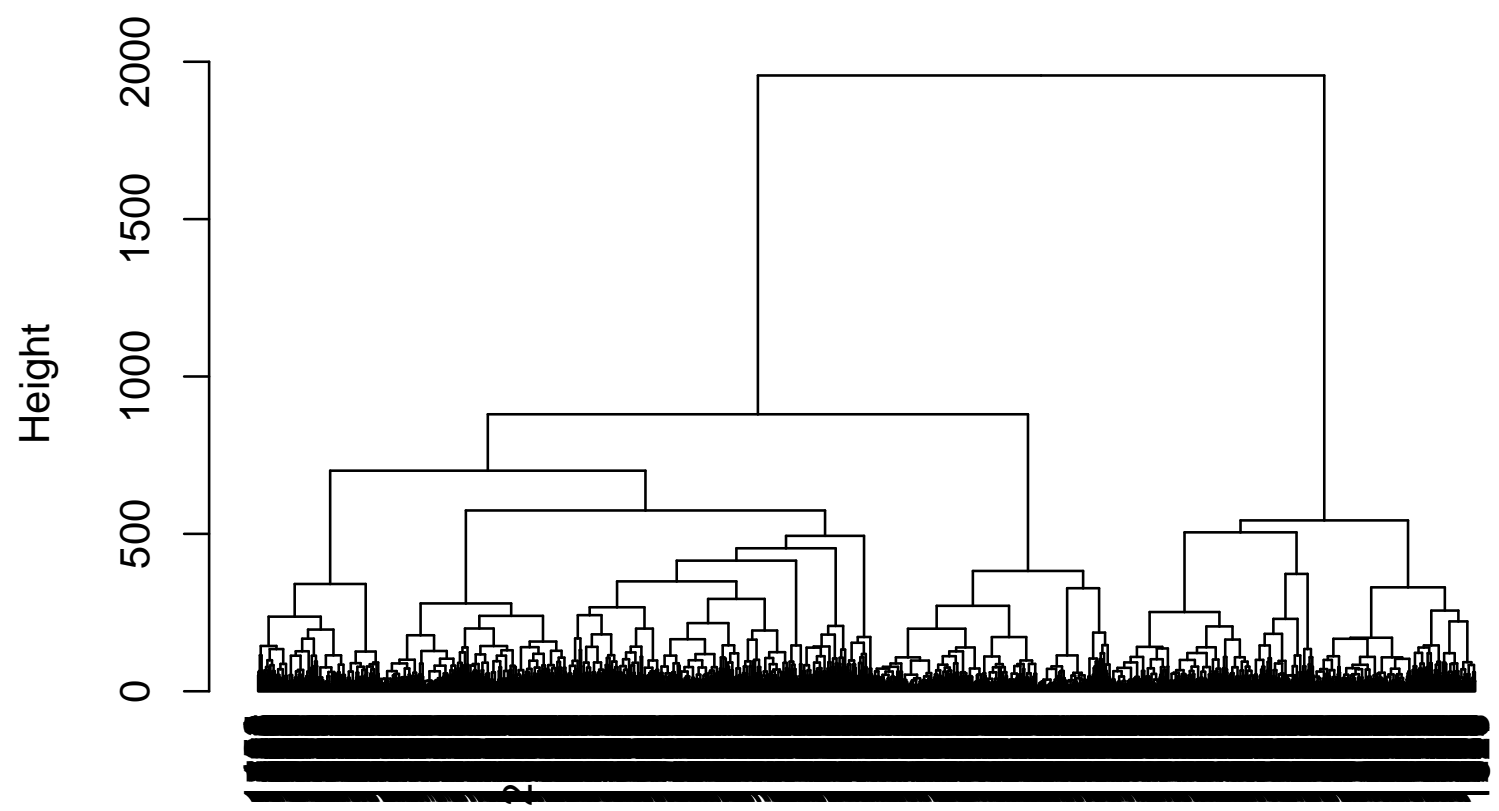

\section{How good is our clustering?}

Let's inspect the splitting tree branches:

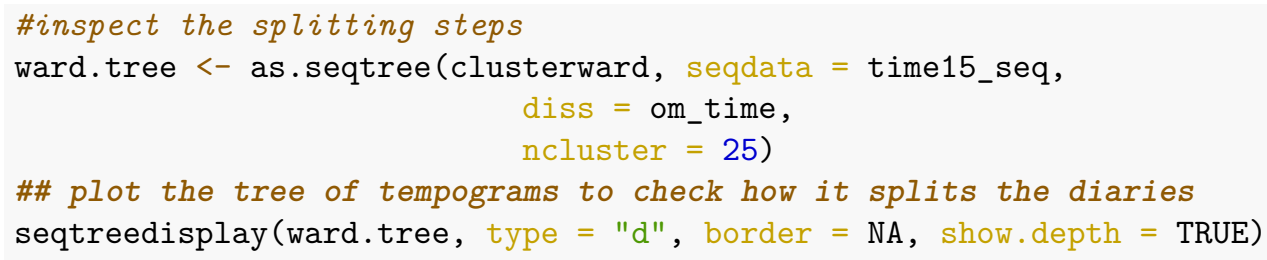

There are many possible tests for the cluster solution quality: - Point Biserial Correlation (PBC). - Hubert's Gamma (HG). - Hubert's Gamma (Somers'D) (HGSD). - Average Silhouette Width (ASW). - Average Silhouette Width (weighted) (ASWw). - Calinski-Harabasz index (CH). - R2. - Calinski-Harabasz Index squared (CHsq). - R2sq. - Hubert's C coefficient (HC).

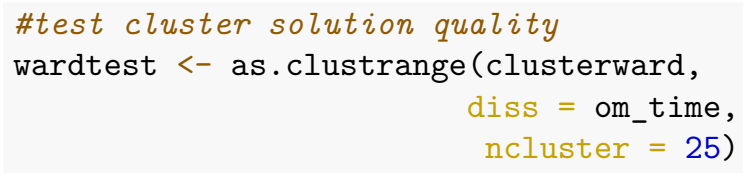




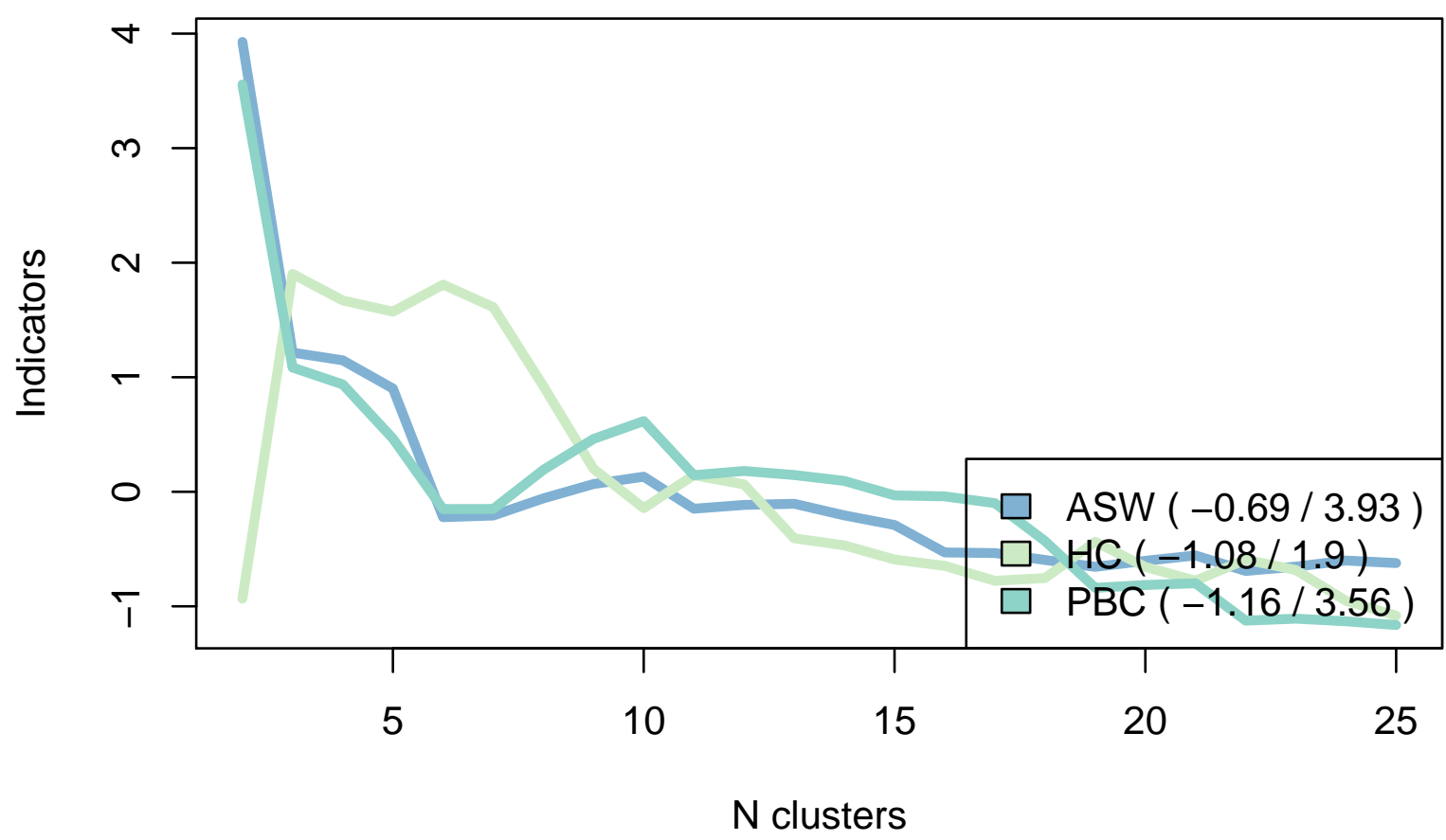

Let's say that our solution is pretty good for 8 clusters.

1. Cut the tree

c8 $<-$ cutree (clusterward, $\mathrm{k}=8$ )

\#\# bind with the dataset

MyData<-cbind (MyData, c8)

2. Plot the cluster solution (will save in the working directory)

\#plot cluster solution

png("plot_clusters.png", 1200, 800)

seqdplot(time15_seq, group $=c 8$, border $=\mathrm{NA}$ )

dev.off ()

\#\# pdf

\#\# 2

\section{How to plot a cluster}

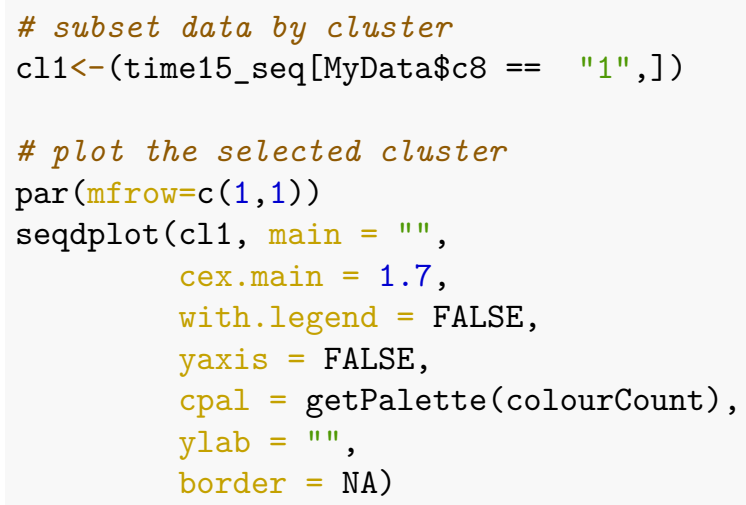




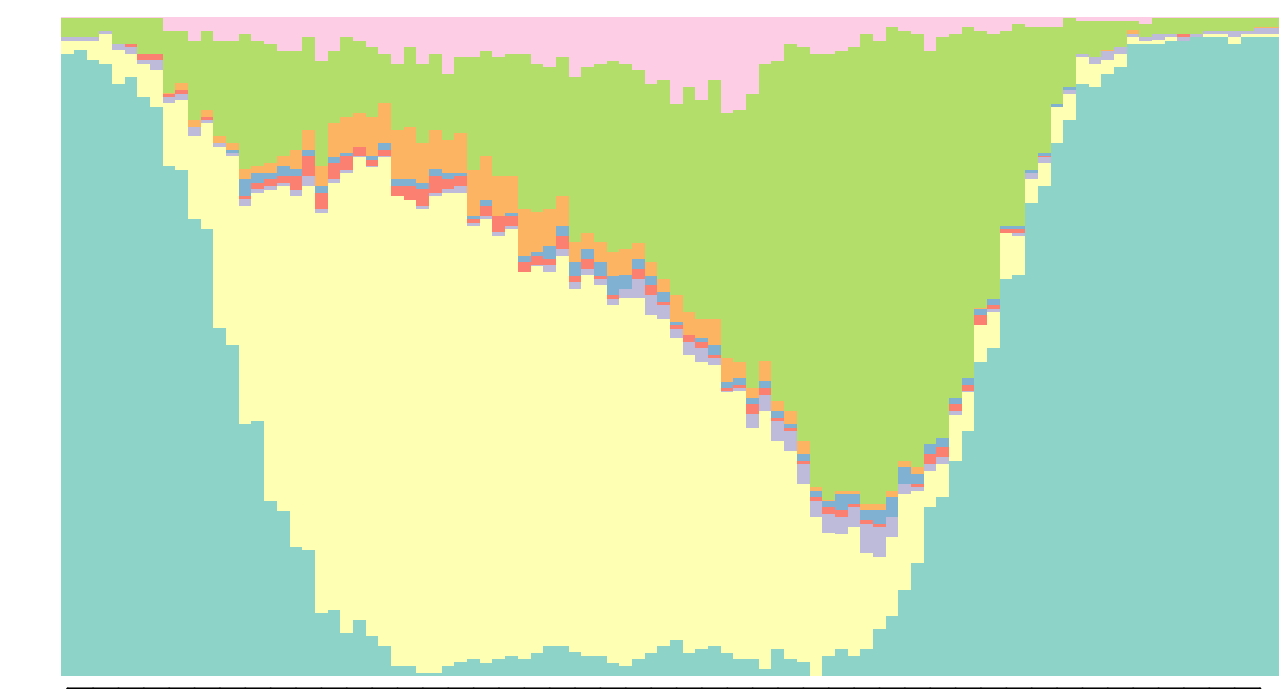

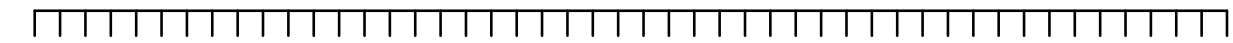

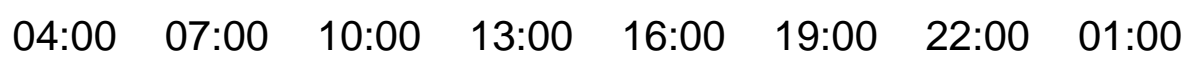

\#\# write new data to csv if needed

\#write.csv(MyData, "clustered_EC.csv") 